\title{
Ferrocene-Terminated Oligourea Foldamer Monolayers
}

Damian Dziubak¹, Karolina Pułka-Ziach*2, Stawomir Sęk*1

${ }^{1}$ Faculty of Chemistry, Biological and Chemical Research Centre, University of Warsaw, Zwirki $i$ Wigury 101, 02-089, Warsaw, Poland.

${ }^{2}$ Faculty of Chemistry, University of Warsaw, Pasteura 1, 02-093 Warsaw, Poland.

- Number of pages: 8

- Number of Figures: 10 


\section{Coupling of $\mathrm{Fc}-\mathrm{COOH}$ and succinimidyl carbamate derivative of cysteamine}

A)

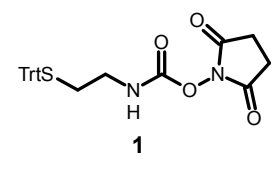

Trt-S-protected cysteamine activated carbamate

B)

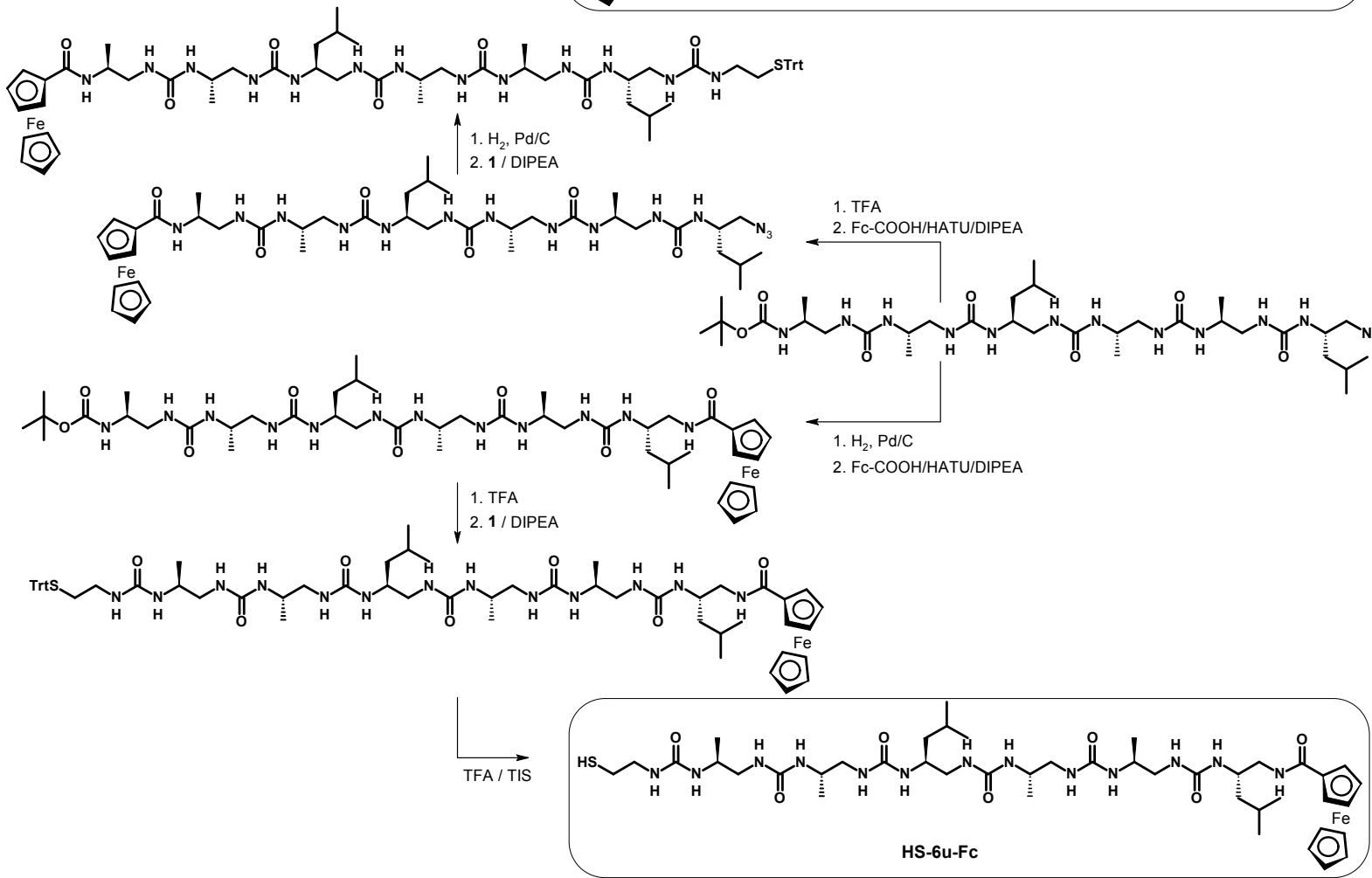

Figure S1. A) the structure of cysteamine building block; B) the scheme of the synthesis of oligourea foldamers with ferrocene and cysteamine moieties

\section{NMR characterization of Fc-6u-SH and HS-6u-Fc}

Experiments were recorded on a Bruker AVANCE $(500 \mathrm{MHz})$ spectrometer at $298 \mathrm{~K}$. Spin systems were assigned using COSY and TOCSY spectra. Sequential assignment was performed on the basis of $\mathrm{N}^{\prime} \mathrm{H}(i+1) / \mathrm{NH}(i)$ ROESY correlations. Compounds were dissolved in $\mathrm{CD}_{3} \mathrm{CN}$ at a concentration $2-3 \mathrm{mM}$. 


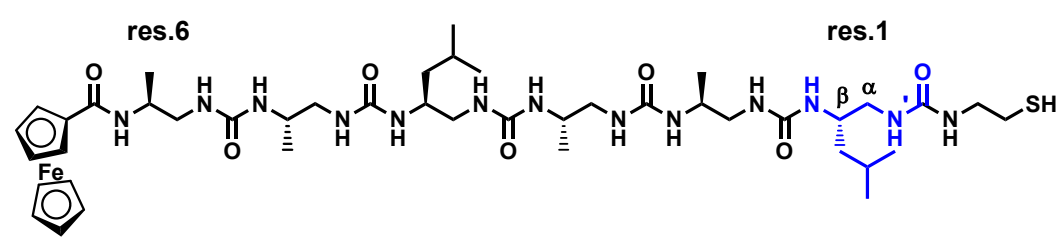

\begin{tabular}{|c|c|c|c|c|c|c|c|c|c|}
\hline Residue & HN & HN' $^{\prime}$ & ${ }^{\alpha} \mathrm{CH}^{1}$ & ${ }^{\alpha} \mathrm{CH}^{2}$ & ${ }^{\beta} \mathrm{CH}$ & $\gamma \mathrm{CH}$ & ${ }^{8} \mathrm{CH}$ & ${ }^{\varepsilon} \mathrm{CH}$ & $\begin{array}{l}\mathrm{Fc} / \text { cysteamine } \\
\text { residues }\end{array}$ \\
\hline Fc & - & - & - & - & - & - & - & - & $\begin{array}{l}4.76, \quad 4.77, \\
4.41,4.19\end{array}$ \\
\hline 6 & $\begin{array}{l}6.60 \\
(\mathrm{~d}, J=10.0 \mathrm{~Hz})\end{array}$ & $\begin{array}{l}5.41 \\
(\mathrm{dd}, J=7.8,4.9 \mathrm{~Hz})\end{array}$ & 3.50 & 2.60 & 4.35 & 1.19 & - & - & - \\
\hline 5 & $\begin{array}{l}4.95 \\
(d, J=10.5 \mathrm{~Hz})\end{array}$ & $\begin{array}{l}6.02 \\
\text { (overlaid with other } \\
\text { signal) }\end{array}$ & 3.59 & 2.35 & 3.84 & 1.01 & - & - & - \\
\hline 4 & $\begin{array}{l}6.19 \\
(\mathrm{~d}, J=10.5 \mathrm{~Hz})\end{array}$ & $\begin{array}{l}6.55 \\
(\mathrm{dd}, J=9.4,4.1 \mathrm{~Hz})\end{array}$ & 3.61 & 2.32 & 3.94 & 1.23 & 1.78 & 0.94 & - \\
\hline 3 & $\begin{array}{l}6.02 \\
\text { (overlaid with } \\
\text { other signal) }\end{array}$ & $\begin{array}{l}6.70 \\
(d d, J=10.2,2.9 \\
H z)\end{array}$ & 3.60 & 2.23 & 3.84 & 0.97 & - & - & - \\
\hline 2 & $\begin{array}{l}5.74 \\
(\mathrm{~d}, J=10.5 \mathrm{~Hz})\end{array}$ & $\begin{array}{l}6.36 \\
(\mathrm{dd}, J=9.9,3.1 \mathrm{~Hz})\end{array}$ & 3.57 & 2.21 & 4.08 & 0.97 & - & - & - \\
\hline 1 & $\begin{array}{l}5.76 \\
(\mathrm{~d}, J=10.0 \mathrm{~Hz})\end{array}$ & $\begin{array}{l}6.30 \\
\text { (overlaid with other } \\
\text { signal) }\end{array}$ & 3.59 & 2.39 & 3.85 & 1.16 & 1.63 & 0.90 & - \\
\hline $\mathrm{HS}\left(\mathrm{CH}_{2}\right)_{2} \mathrm{NH}$ & $\begin{array}{l}6.32 \\
\text { (overlaid with } \\
\text { other signal) }\end{array}$ & - & - & - & - & - & - & - & $\begin{array}{l}3.32\left(\mathrm{CH}_{2} \mathrm{NH}\right), \\
3.17\left(\mathrm{CH}_{2} \mathrm{NH}\right), \\
2.56\left(\mathrm{CH}_{2} \mathrm{SH}\right), \\
1.91(\mathrm{SH})\end{array}$ \\
\hline
\end{tabular}




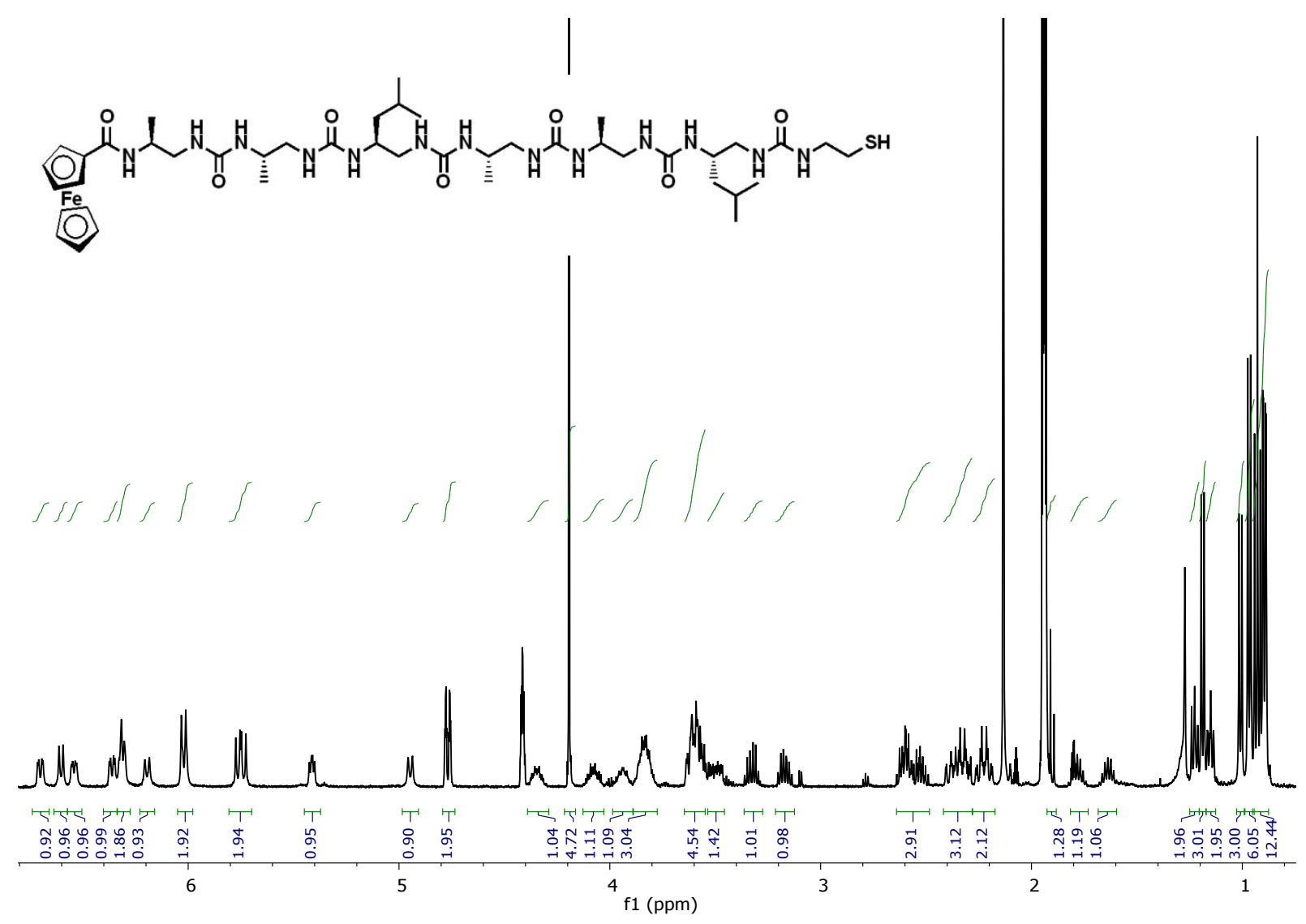

Figure S2. 1H NMR of compound Fc-6u-SH $\left(\mathrm{CD}_{3} \mathrm{CN}, 500 \mathrm{MHz}\right)$

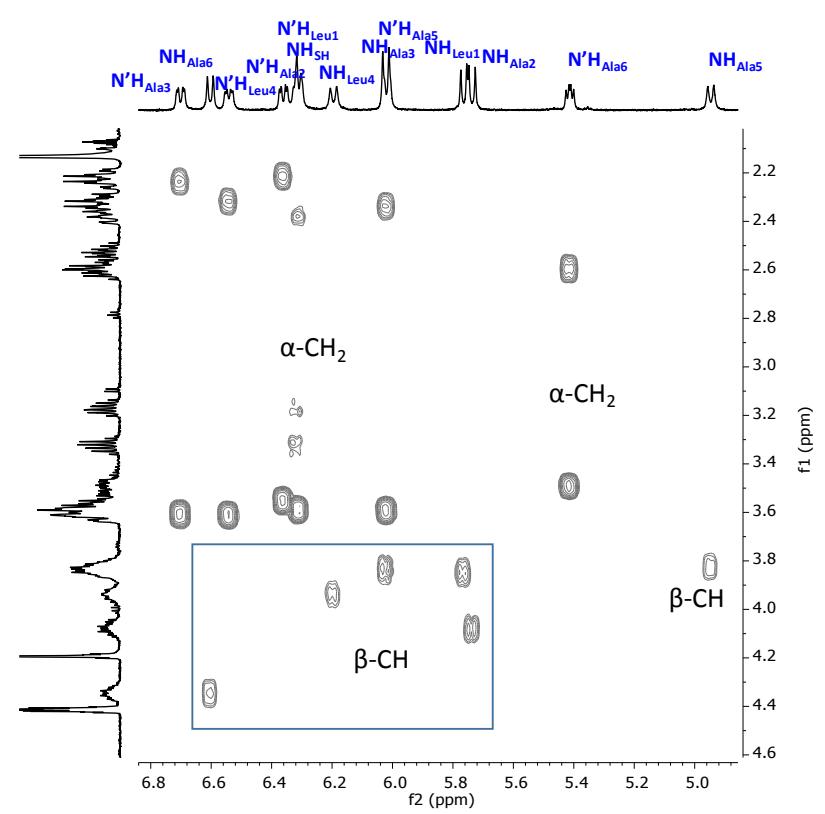

Figure S3. Fingerprint $\mathrm{NH} / \beta-\mathrm{CH}$ and $\mathrm{N}^{\prime} \mathrm{H} / \alpha-\mathrm{CH}$ region of the COSY spectrum of compound Fc-6u-SH 


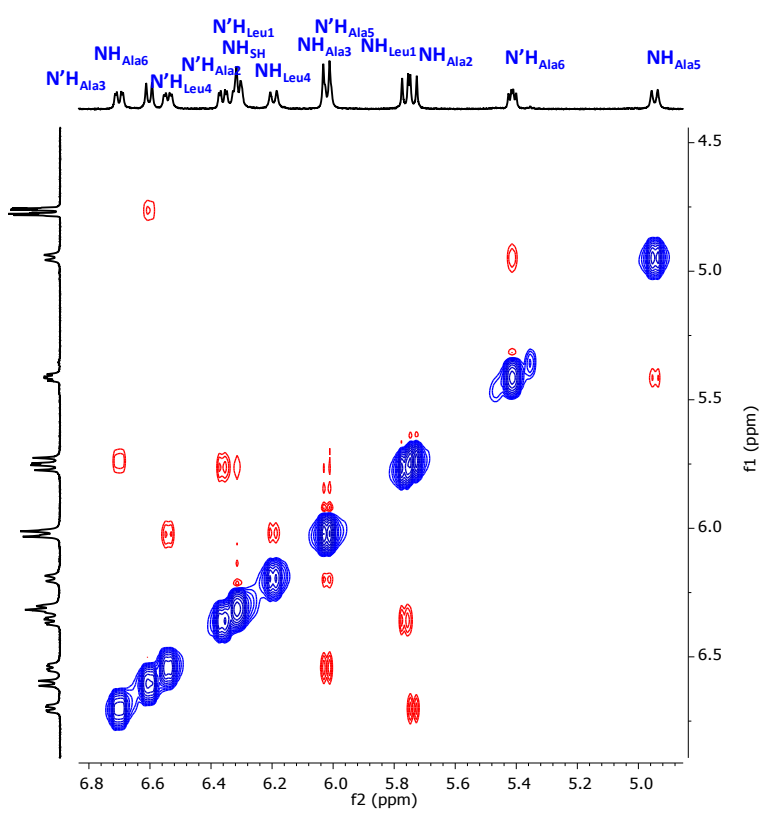

Figure S4. ROESY spectrum with ROE cross peaks showing the transfer of nuclear spin polarisation within urea group $\left(\mathrm{NH} / \mathrm{N}^{\prime} \mathrm{H}\right)$ of compound Fc-6u-SH

HS-6u-Fc

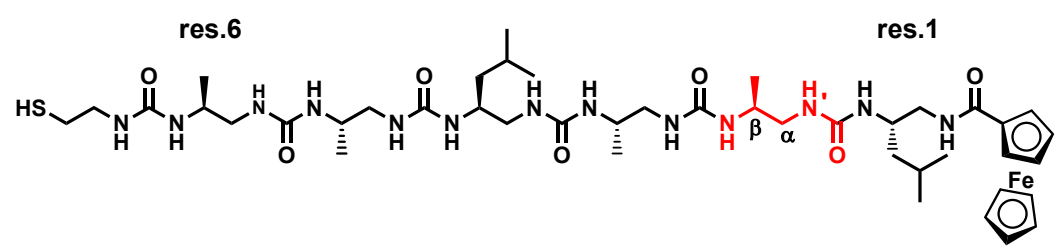

\begin{tabular}{|c|c|c|c|c|c|c|c|c|c|}
\hline Residue & $\mathrm{HN}$ & HN' $^{\prime}$ & ${ }^{\alpha} \mathrm{CH}^{1}$ & ${ }^{\alpha} \mathrm{CH}^{2}$ & ${ }^{\beta} \mathrm{CH}$ & ${ }^{\gamma} \mathrm{CH}$ & ${ }^{8} \mathrm{CH}$ & ${ }^{\varepsilon} \mathbf{C H}$ & $\begin{array}{l}\text { Fc/cysteamine } \\
\text { residues }\end{array}$ \\
\hline $\mathrm{HS}\left(\mathrm{CH}_{2}\right)_{2} \mathrm{NH}$ & $\begin{array}{l}5.42 \\
\text { (overlaid with } \\
\text { other signal) }\end{array}$ & - & - & - & - & - & - & - & $\begin{array}{l}3.30\left(\mathrm{CH}_{2} \mathrm{NH}\right), \\
2.60\left(\mathrm{CH}_{2} \mathrm{SH}\right), \\
1.65(\mathrm{SH})\end{array}$ \\
\hline 6 & $\begin{array}{l}5.07 \\
(d, J=9.5 \mathrm{~Hz})\end{array}$ & $\begin{array}{l}5.42 \\
\text { (overlaid with other } \\
\text { signal) }\end{array}$ & 3.44 & 2.42 & 3.95 & 1.03 & - & - & - \\
\hline 5 & $\begin{array}{l}5.00 \\
(d, J=10.0 \mathrm{~Hz})\end{array}$ & $\begin{array}{l}6.17 \\
\text { (overlaid with other } \\
\text { signal) }\end{array}$ & 3.59 & 2.33 & 3.84 & 1.03 & - & - & - \\
\hline 4 & $\begin{array}{l}6.00 \\
(\mathrm{~d}, J=10.7 \mathrm{~Hz})\end{array}$ & $\begin{array}{l}6.53 \\
\text { (overlaid with other } \\
\text { signal) }\end{array}$ & 3.57 & 2.24 & 3.89 & 1.16 & 1.68 & 0.89 & - \\
\hline 3 & $\begin{array}{l}5.96 \\
(d, J=10.4 \mathrm{~Hz})\end{array}$ & $\begin{array}{l}6.53 \\
\text { (overlaid with other } \\
\text { signal) }\end{array}$ & 3.60 & 2.20 & 3.99 & 0.96 & - & - & - \\
\hline 2 & $\begin{array}{l}5.70 \\
(d, J=10.2 \mathrm{~Hz})\end{array}$ & $\begin{array}{l}6.20 \\
\text { (overlaid with other } \\
\text { signal) }\end{array}$ & 3.59 & 2.31 & 4.07 & 0.98 & - & - & - \\
\hline 1 & $\begin{array}{l}6.05 \\
(d, J=9.1 \mathrm{~Hz})\end{array}$ & $\begin{array}{l}7.89 \\
(\mathrm{dd}, J=7.1,3.8 \mathrm{~Hz})\end{array}$ & 3.78 & 2.77 & 3.86 & 1.27 & 1.72 & 0.92 & - \\
\hline $\mathrm{Fc}$ & & & & & & & & & $\begin{array}{l}4.96, \quad 4.91, \\
4.28,4.20\end{array}$ \\
\hline
\end{tabular}




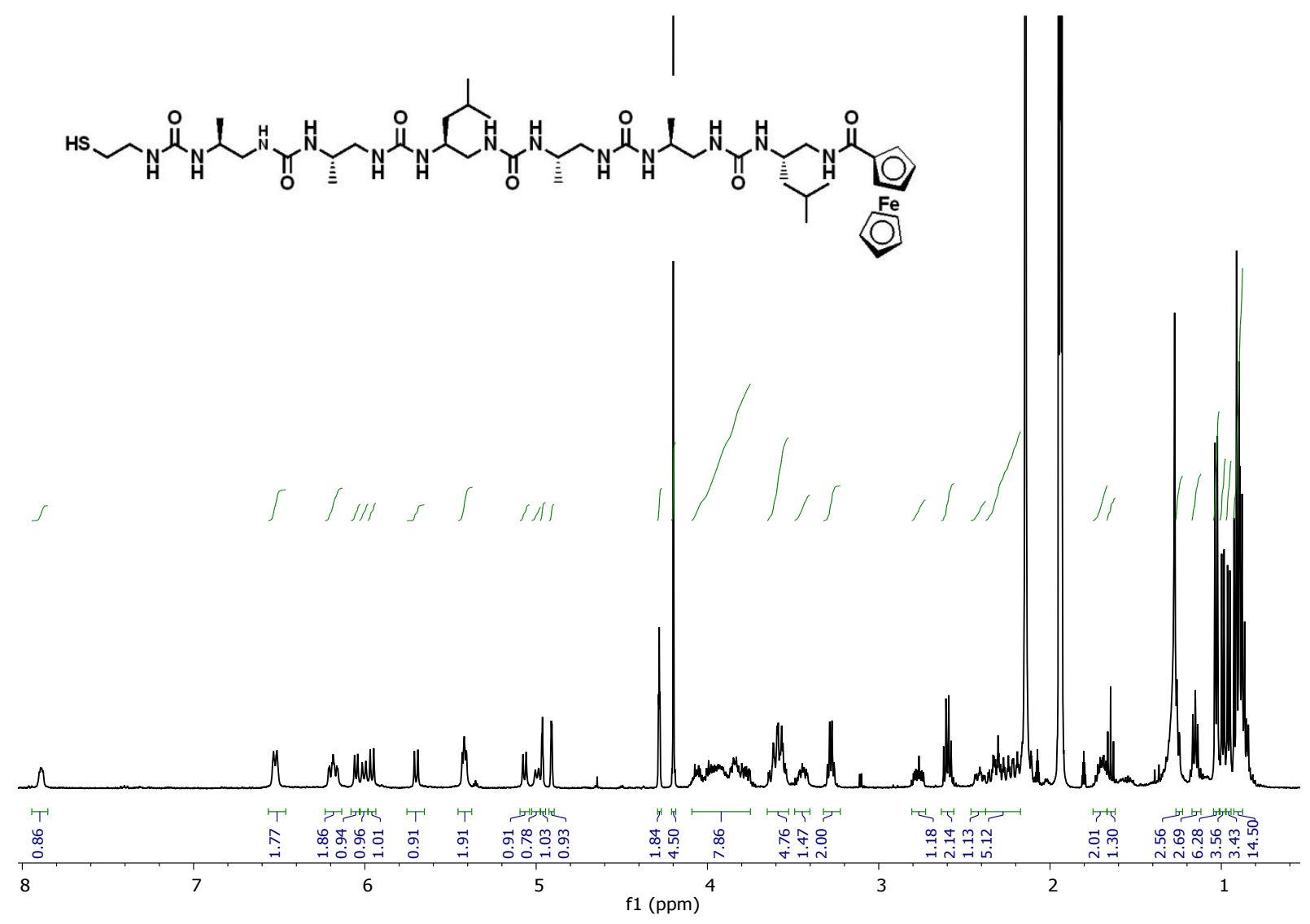

Figure S5. $1 \mathrm{H}$ NMR of compound HS-6u-Fc $\left(\mathrm{CD}_{3} \mathrm{CN}, 500 \mathrm{MHz}\right)$

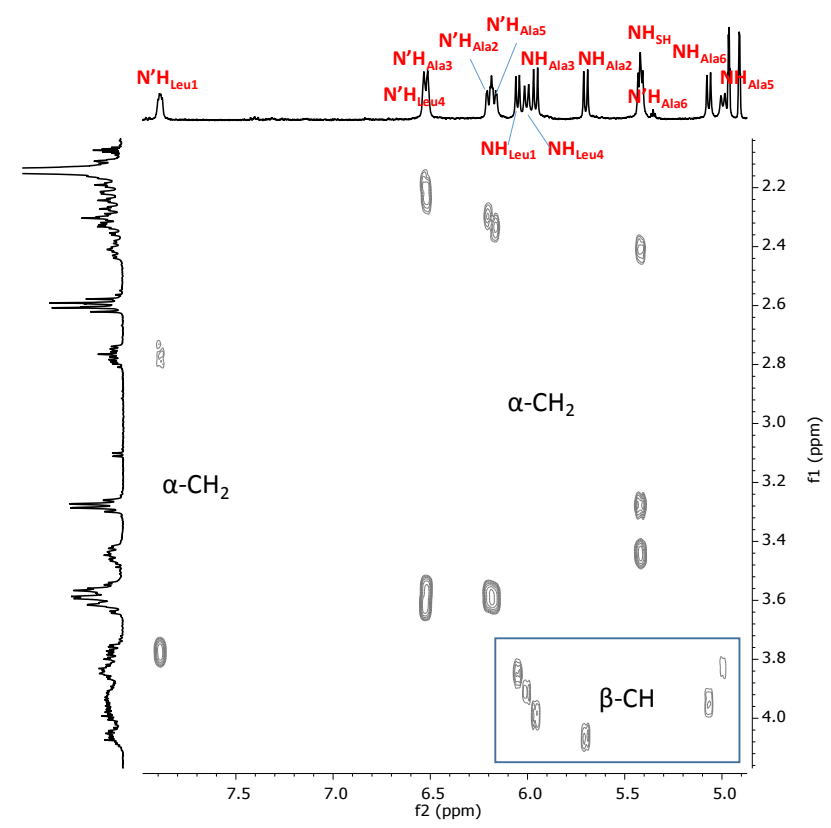

Figure S6. Fingerprint $\mathrm{NH} / \beta-\mathrm{CH}$ and $\mathrm{N}^{\prime} \mathrm{H} / \alpha-\mathrm{CH}$ region of the COSY spectrum of compound $\mathrm{Fc}-6 \mathrm{u}-\mathrm{SH}$ 


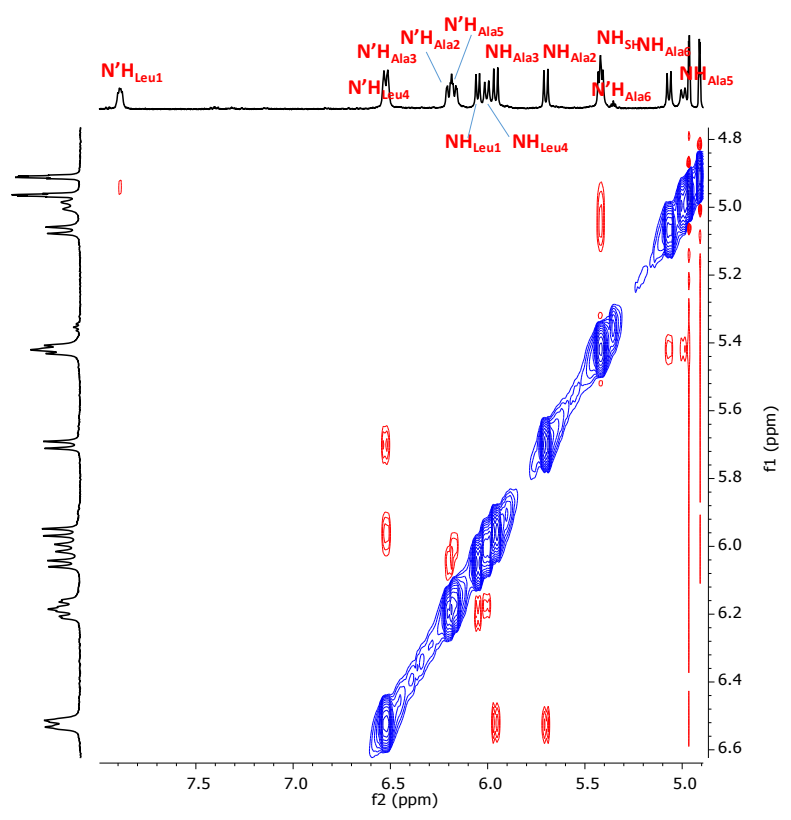

Figure S7. ROESY spectrum with ROE cross peaks showing the transfer of nuclear spin polarisation within urea group $\left(\mathrm{NH} / \mathrm{N}^{\prime} \mathrm{H}\right)$ of compound $\mathrm{HS}-6 \mathrm{u}-\mathrm{Fc}$

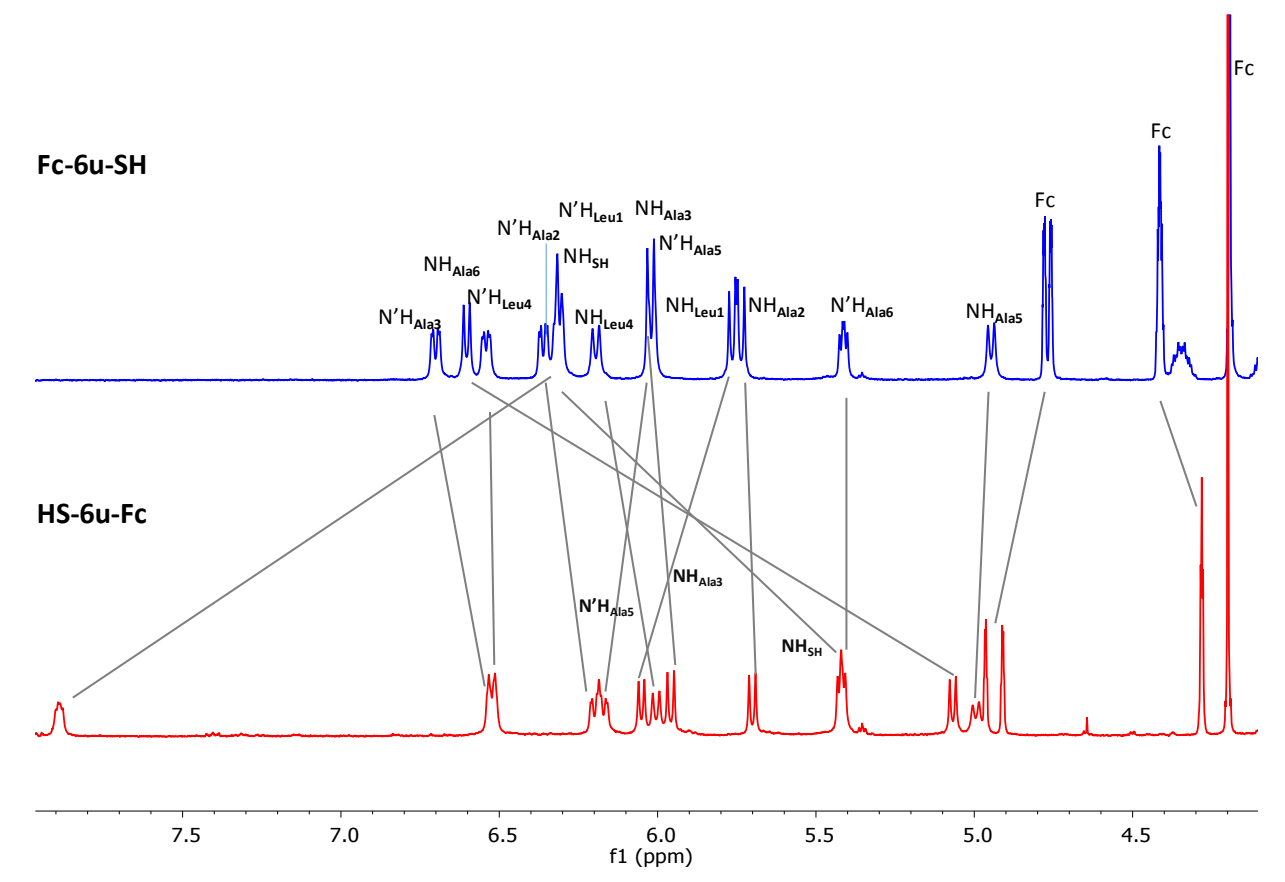

Figure S8. Comparison of the region of urea protons of $1 \mathrm{H}$ spectra of Fc-6u-SH and HS-6u-Fc 
3. UV-Vis and CD spectra of Fc-6u-SH and HS-6u-Fc
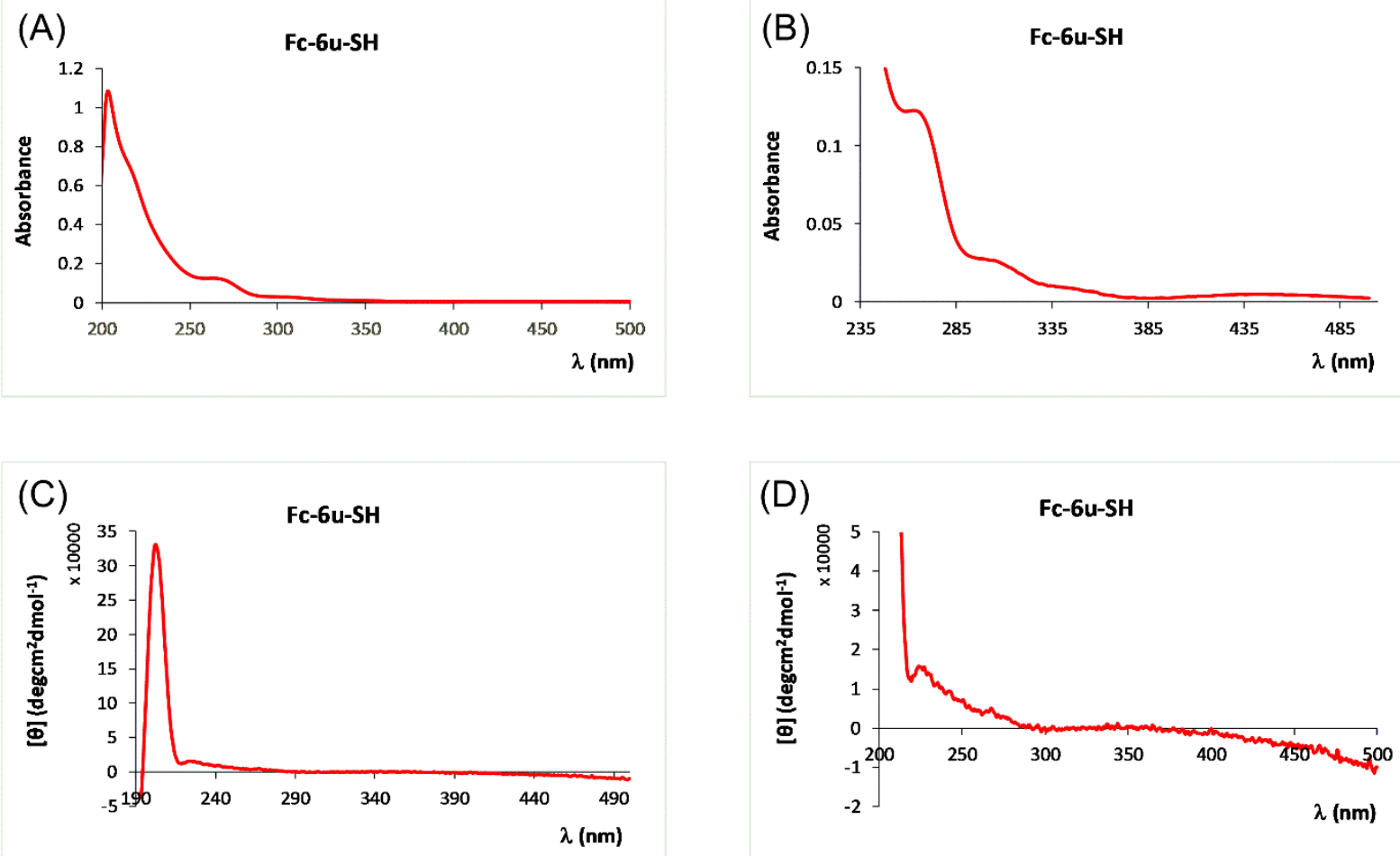

Figure S 9. Spectra of compound Fc-6u-SH: UV-Vis spectra (c=2.1× $\left.10^{-5} \mathrm{M}\right)$ A) $\left.\lambda=200-500 \mathrm{~nm}, \mathrm{~B}\right) \lambda=$ $235-500 \mathrm{~nm} ; \mathrm{CD}$ spectra $\left.\left.\left(\mathrm{c}=2.1 \times 10^{-4} \mathrm{M}\right) \mathrm{C}\right) \lambda=193-500 \mathrm{~nm}, \mathrm{D}\right) \lambda=200-500 \mathrm{~nm}$
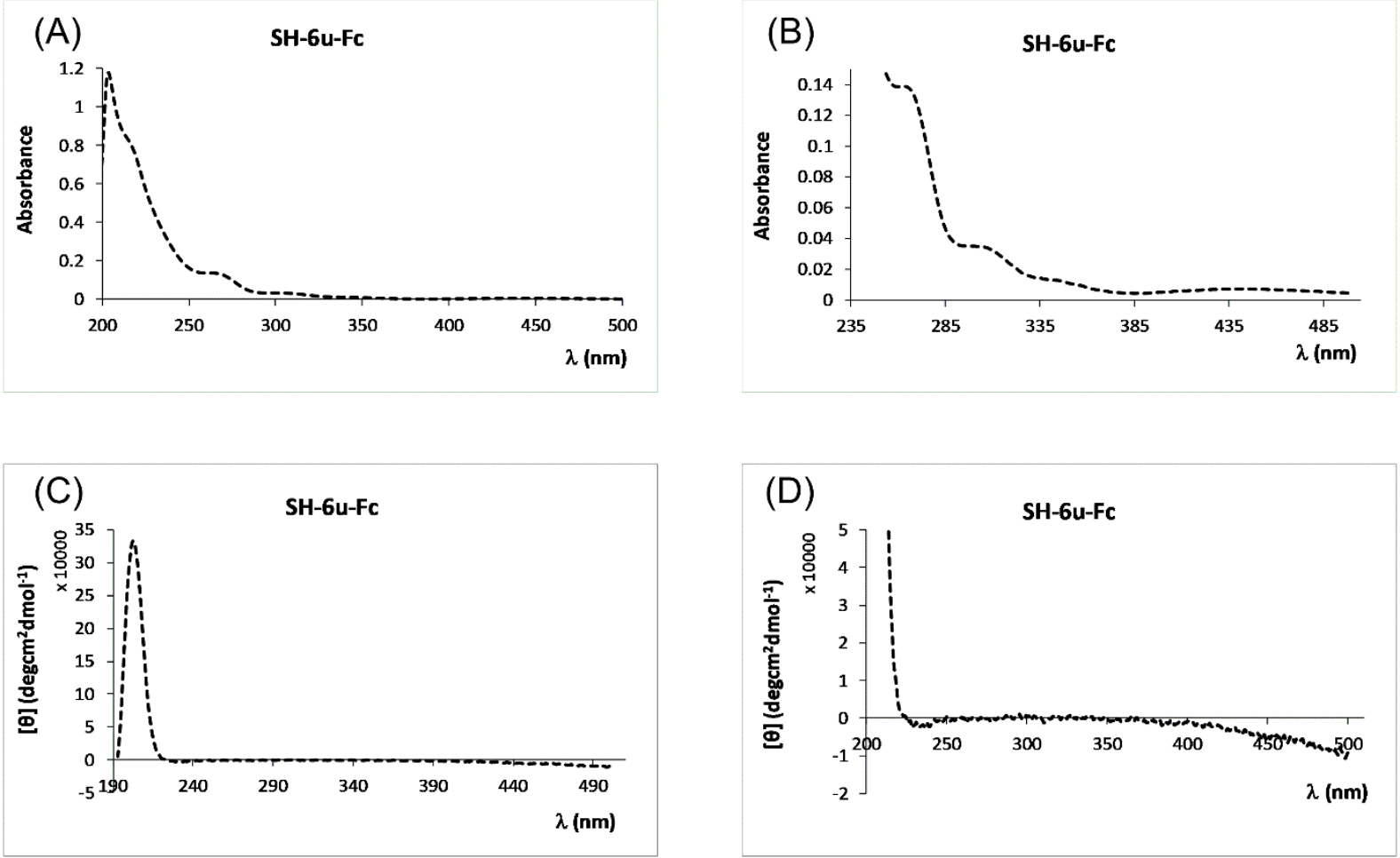

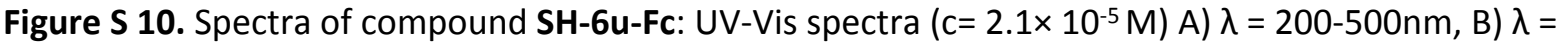
$235-500 \mathrm{~nm}$; CD spectra $\left(\mathrm{c}=2.1 \times 10^{-4}\right.$ M) C) $\left.\lambda=193-500 \mathrm{~nm}, \mathrm{D}\right) \lambda=200-500 \mathrm{~nm}$ 